University of Nebraska - Lincoln

DigitalCommons@University of Nebraska - Lincoln

9-23-2002

\title{
Domain Wall Relaxation, Creep, Sliding, and Switching in Superferromagnetic Discontinuous $\mathrm{Co}_{80} \mathrm{Fe}_{20} / \mathrm{Al}_{2} \mathrm{O}_{3}$ Multilayers
}

\author{
X. Chen \\ Laboratorium fu“ r Angewandte Physik, Gerhard-Mercator-Universita"t, Duisburg, Duisburg, Germany \\ O. Sichelschmidt \\ Laboratorium fu" r Angewandte Physik, Gerhard-Mercator-Universita"t, Duisburg, Duisburg, Germany \\ Wolfgang Kleemann \\ Laboratorium fu" r Angewandte Physik, Gerhard-Mercator-Universita"t, Duisburg, Duisburg, Germany, \\ wolfgang.kleemann@uni-due.de \\ Christian Binek \\ University of Nebraska-Lincoln, cbinek@unl.edu \\ J.B. Sousa \\ IFIMUP, Departamento de Fisica, Universidade de Porto, Porto, Portugal \\ See next page for additional authors
}

Follow this and additional works at: https://digitalcommons.unl.edu/physicsbinek

Part of the Physics Commons

Chen, X.; Sichelschmidt, O.; Kleemann, Wolfgang; Binek, Christian; Sousa, J.B.; Cardoso de Freitas, Susana; and Freitas, P.P., "Domain Wall Relaxation, Creep, Sliding, and Switching in Superferromagnetic Discontinuous $\mathrm{Co}_{80} \mathrm{Fe}_{20} / \mathrm{Al}_{2} \mathrm{O}_{3}$ Multilayers" (2002). Christian Binek Publications. 20.

https://digitalcommons.unl.edu/physicsbinek/20

This Article is brought to you for free and open access by the Research Papers in Physics and Astronomy at DigitalCommons@University of Nebraska - Lincoln. It has been accepted for inclusion in Christian Binek Publications by an authorized administrator of DigitalCommons@University of Nebraska - Lincoln. 
Authors

X. Chen, O. Sichelschmidt, Wolfgang Kleemann, Christian Binek, J.B. Sousa, Susana Cardoso de Freitas, and P.P. Freitas

This article is available at DigitalCommons@University of Nebraska - Lincoln: https://digitalcommons.unl.edu/ physicsbinek/20 


\title{
Domain Wall Relaxation, Creep, Sliding, and Switching in Superferromagnetic Discontinuous $\mathrm{Co}_{80} \mathrm{Fe}_{20} / \mathrm{Al}_{2} \mathrm{O}_{3}$ Multilayers
}

\author{
X. Chen, ${ }^{1}$ O. Sichelschmidt, ${ }^{1}$ W. Kleemann, ${ }^{1}$ O. Petracic, ${ }^{1}$ Ch. Binek, ${ }^{1}$ J. B. Sousa, ${ }^{2}$ S . Cardoso, ${ }^{3}$ and P. P. Freitas ${ }^{3}$ \\ ${ }^{1}$ Laboratorium für Angewandte Physik, Gerhard-Mercator-Universität, Duisburg, D-47048 Duisburg, Germany \\ ${ }^{2}$ IFIMUP, Departamento de Fisica, Universidade de Porto, 4169-007 Porto, Portugal \\ ${ }^{3}$ INESC, Rua Alves Redol 9-1, 1000 Lisbon, Portugal \\ (Received 9 April 2002; published 9 September 2002)
}

\begin{abstract}
The ac susceptibility of a superferromagnetic discontinuous multilayer $\left[\mathrm{Co}_{80} \mathrm{Fe}_{20}(1.4 \mathrm{~nm}) /\right.$ $\left.\mathrm{Al}_{2} \mathrm{O}_{3}(3 \mathrm{~nm})\right]_{10}$ is measured as a function of temperature, frequency, and field amplitude and compared to static and dynamic hysteresis loops. Its properties are successfully mapped onto the predicted [T. Nattermann, V. Pokrovsky, and V. M. Vinokur, Phys. Rev. Lett. 87, 197005 (2001).] dynamical phase transitions, which link the relaxation, creep, sliding, and switching regimes of pinned domain walls.
\end{abstract}

DOI: $10.1103 /$ PhysRevLett.89.137203

Recently nanosized magnetic single domain particles ("superspins") and their mutual interactions have attracted much interest both in fundamental research and applications. A novel type of superferromagnetic (SFM) behavior was discovered in discontinuous metal-insulator multilayer (DMIM) systems such as $\left[\mathrm{Co}_{80} \mathrm{Fe}_{20}(t) /\right.$ $\left.\mathrm{Al}_{2} \mathrm{O}_{3}(3 \mathrm{~nm})\right]_{10}$ [1]. It occurs at nominal CoFe thicknesses $t>1.2 \mathrm{~nm}$, but below the percolation limit, $t_{p}=1.8 \mathrm{~nm}$, above which a normal 3D ferromagnetic phase with Ohmic conduction is encountered [2]. A similar nonpercolating SFM state of Co islands was observed in $\mathrm{Co}(t) / \mathrm{Cu}(100)$ thin films at coverages $t<1.8$ monolayers [3]. Monte Carlo simulations show that the dipolarly coupled islands attain a nondegenerate noncollinear ground state [4]. However, in view of clear ferromagnetic signatures (hysteresis and stable remanence $[1,3]$ ), very probably additional nonclassic ferromagnetic interactions are involved [5].

Although details of the SFM superspin structure are not yet known, it is clear that any field-induced change of its "order-by-disorder" [6] will compete with inherent random bonds and random anisotropy. Indeed, as shown in this Letter, the SFM state of the DMIM system $\left[\mathrm{Co}_{80} \mathrm{Fe}_{20}(1.4 \mathrm{~nm}) / \mathrm{Al}_{2} \mathrm{O}_{3}(3 \mathrm{~nm})\right]_{10}$ strongly reminds one of an impure magnet containing a broad distribution of random field or random bondlike pinning centers for domain walls. Analysis of its complex susceptibility, $\chi^{*}=\chi^{\prime}-i \chi^{\prime \prime}$, as a function of $T$, frequency $f=\omega / 2 \pi$ and probing ac field amplitude, $h_{0}$, evidences fundamental dynamic features predicted for the dynamic hysteresis of an impure magnet containing one domain wall [7].

When increasing $h_{0}$, the magnetization $(M)$-versus-magnetic field $(h)$ loop displays four regimes linked by dynamical phase transitions. At very low fields, $h_{0}<h_{\omega}$, only relaxation, but no macroscopic motion of the walls should occur at finite frequencies. Within the range $h_{\omega}<h_{0}<h_{t 1}$, a thermally activated "creep" is expected, while above the depinning threshold $h_{t 1}$ the "sliding" regime is encountered within $h_{t 1}<h_{0}<h_{t 2}$.
PACS numbers: 75.40.Gb, 75.60.Ej, 75.70.Cn, 78.20.Ls

Finally, for $h_{0}>h_{t 2}$, a complete reversal of the magnetization (switching) occurs in the whole sample in each half of the period, $\tau=1 / f$. It should be noticed that all transition fields, $h_{\omega}, h_{t 1}$, and $h_{t 2}$, are expected to depend strongly on both $T$ and $\omega$ [7].

The DMIM sample of $\left[\mathrm{Co}_{80} \mathrm{Fe}_{20}(1.4 \mathrm{~nm}) /\right.$ $\left.\mathrm{Al}_{2} \mathrm{O}_{3}(3 \mathrm{~nm})\right]_{10}$ was prepared by focused ion beam sputtering as described previously [2]. Owing to pronounced Volmer-Weber-type growth, the nonwetting $\mathrm{Co}_{80} \mathrm{Fe}_{20}$ alloy forms nearly spherical granules in the amorphous $\mathrm{Al}_{2} \mathrm{O}_{3}$ matrix similarly as experienced previously on Co-based DMIMs [8]. A field-induced uniaxial in-plane anisotropy defines the easy directions of both the ac susceptibility measured at frequencies $10^{-2} \leq f \leq$ $10^{3} \mathrm{~Hz}$ and temperatures $200 \leq T \leq 380 \mathrm{~K}$ and the dc magnetization measured at variant fields with a superconducting quantum interference device (SQUID) magnetometer (Quantum Design MPMS-5S). Prior to each measurement, flux gate controlled zero-field conditions were achieved to within $\left|\mu_{0} H\right|<2 \mu \mathrm{T}$ by quenching the superconducting solenoid and compensating the remanent field. Dynamic hysteresis cycles were measured via the longitudinal magneto-optical Kerr effect (MOKE) in sinusoidal fields, $h=h_{0} \exp (i \omega t)$, with $\mu_{0} h_{0}=6 \mathrm{mT}$ at $0.002 \leq f \leq 80 \mathrm{~Hz}$.

Apart from its large polydispersivity [1], the ac susceptibility surprises by its extreme nonlinearity as shown in Fig. 1 for two field amplitudes, $\mu_{0} h_{0}=50$ and $200 \mu \mathrm{T}$, and frequencies $0.01 \leq f \leq 1 \mathrm{~Hz}$. An increase of $\mu_{0} h_{0}$ by a factor of 4 causes the peaks of $\chi^{\prime}\left(\chi^{\prime \prime}\right)$ vs $T$ to shift to lower $T$ by 30(50) $\mathrm{K}$ and to shrink by factors of 5(3). Simultaneously, their profiles change appreciably unlike the corresponding curves of a DMIM with typical superspin glass (SSG) properties as observed in the low "concentration range," $t \leq 1.0 \mathrm{~nm}[1,9]$.

The unusual appearance of SFM polydispersivity is most clearly recognized in so-called Cole-Cole diagrams, $\chi^{\prime \prime}$ vs $\chi^{\prime}$ [10], as shown in Fig. 2 for $T=$ 380 (1), 350 (2), 320 (3), and 260 K (4), with 


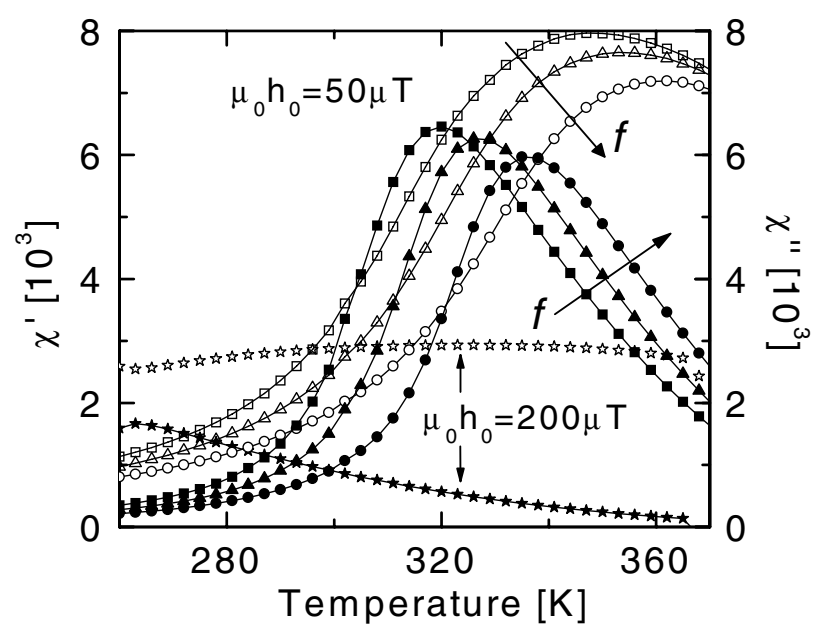

FIG. 1. Temperature dependence of the in-plane susceptibility $\chi^{\prime}$ (open symbols) and $\chi^{\prime \prime}$ (solid symbols) of $\left[\mathrm{Co}_{80} \mathrm{Fe}_{20}(1.4 \mathrm{~nm}) / \mathrm{Al}_{2} \mathrm{O}_{3}(3 \mathrm{~nm})\right]_{10}$ taken with ac amplitudes $\mu_{0} h_{0}=50 \mu \mathrm{T}$ at $f=0.01,0.1$, and $1 \mathrm{~Hz}$ (see arrows and symbol codes), and with $\mu_{0} h_{0}=200 \mu \mathrm{T}$ at $f=0.01 \mathrm{~Hz}$. Solid lines are guides to the eye.

$\mu_{0} h_{0}=50[2(\mathrm{a})]$ and $5 \mu \mathrm{T}[2(\mathrm{~b})]$. At highest frequencies, $f \rightarrow 1 \mathrm{kHz}$ (i.e., $\chi^{\prime} \rightarrow 0$ ), the diagrams start either with proportionalities, $\chi^{\prime \prime} \propto \chi^{\prime}$, or with a horizontal spectrum, $\chi^{\prime \prime} \approx$ const [Figs. 2(a) and 2(b); broken lines]. At increasing $\chi^{\prime}$, the slope $d \chi^{\prime \prime} / d \chi^{\prime}$ first increases andafter passing a point of inflexion and a peak-the diagrams finally convert into classic Cole-Cole semicircles as $f \rightarrow 10 \mathrm{mHz}$ (broken lines fitted to curves 1 and 2), thus revealing monodispersivity [10]. In the following, we first argue that the observed dispersion of $\chi^{*}$ is solely due to domain wall processes. Second, we attribute characteristic features designated by $h_{\omega}$ in Fig. 2(b) (vertical

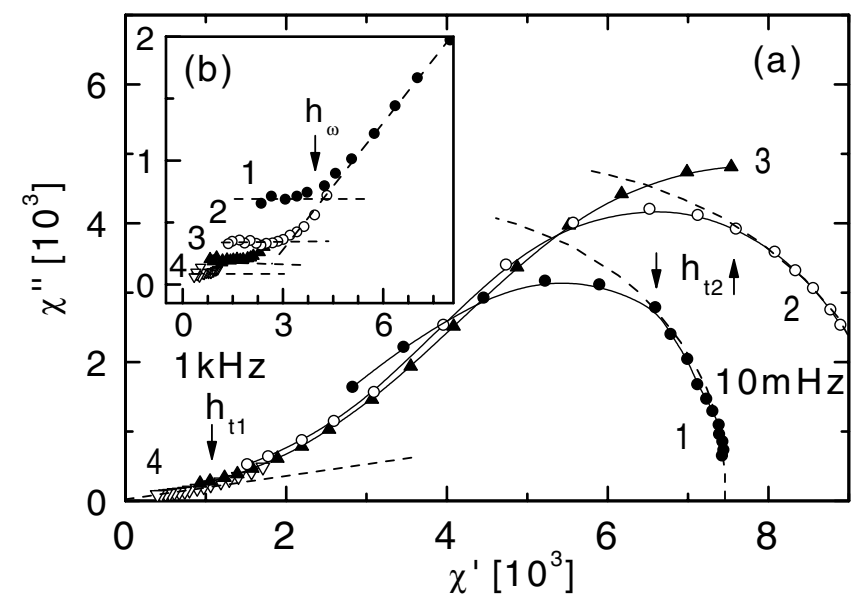

FIG. 2. Susceptibility components $\chi^{\prime \prime}$ vs $\chi^{\prime}$ measured at ac amplitudes $\mu_{0} h_{0}=50$ (a) and $5 \mu \mathrm{T}$ (b) at $10 \mathrm{mHz} \leq f \leq$ $1 \mathrm{kHz}$ (data points from right to left, connected by interpolating solid lines) at $T=380$ (1), 350 (2), 320 (3), and $260 \mathrm{~K}$ (4). Phase transition features (see text) are marked by broken lines. arrows) and by $h_{t 1}$ and $h_{t 2}$ in Fig. 2(a) to dynamic phase transitions predicted for the impure ferromagnet [7].

In order to evidence that the ac susceptometry is controlled by domain wall processes, we inspect the static and dynamic magnetization curves at various temperatures, $270 \leq T \leq 370 \mathrm{~K}$ (Fig. 3), and frequencies, $5 \times$ $10^{-5} \mathrm{~Hz} \lesssim f \leq 80 \mathrm{~Hz}$ (Fig. 4), respectively. From both figures, it is seen that very low fields, $\left|\mu_{0} h\right| \leq 2 \mathrm{mT}$, suffice to switch the magnetization. Hence, susceptometry with $5 \leq \mu_{0} h_{0} \leq 200 \mu \mathrm{T}$ as applied in the present study probes partial hysteresis loops, which-in strong dependence on $h_{0}, T$, and $f$-range from minor loops to complete switching cycles, thus passing all of the predicted dynamical critical fields [7].

In Fig. 3 , it is seen that both coercive field, $H_{c}$, and remanence, $M_{r}$, decrease monotonically as $T$ increases, the latter reaching zero at $T_{c} \approx 407 \mathrm{~K}$. Rounded hysteresis loops with ratios $M_{r} / M_{s} \approx 0.5 \quad\left(M_{s}=\right.$ saturation magnetization) indicate soft ferromagnetism, which demagnetizes in zero field via domain formation as in permalloy or $\mu$ metal. However, the very slow powerlaw-like temporal relaxation of the remanence observed after field cooling to below $T_{c}$ predicts astronomic decay times, e.g., $\Delta t \approx 10^{33} \mathrm{~s}$ for the $90 \%$ decay of $M_{r} \propto t^{-0.03}$ as determined within $10 \leq t \leq 10^{4} \mathrm{~s}$ at $T=320 \mathrm{~K}$.

The dynamical stabilization of the hysteresis is verified by dynamic MOKE loops as shown in Fig. 4 for $T=$ 294 K. As expected [11], the area of the hysteresis loop and the coercive field $h_{c}$ increase as the frequency of magnetization reversal is increased. We find a surprisingly large factor of about 50 between $f \approx 5 \times 10^{-5} \mathrm{~Hz}$ (SQUID data) and $80 \mathrm{~Hz}$. As in conventional ferromagnetic ultrathin films, e.g., $\mathrm{Fe} / \mathrm{GaAs}$ [12], we observe two dynamical regimes obeying power laws, $h_{c} \propto(d H / d t)^{\alpha}$, with different exponents $\alpha$, where $\alpha \approx 0.02 \pm 0.07$ in the "low dynamic regime," $f<0.05 \mathrm{~Hz}$, and $\alpha \approx 0.49 \pm$ 0.04 in the "high dynamic regime," $f>1 \mathrm{~Hz}$ (Fig. 4,

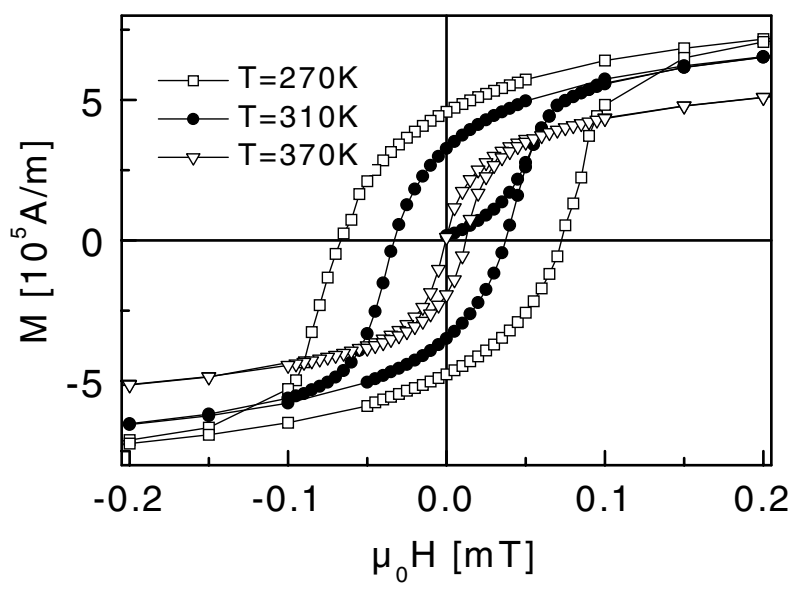

FIG. 3. Static magnetization curves measured by SQUID techniques at $T=270,310$ (complemented by a zero-field cooled virgin curve), and $370 \mathrm{~K}$. 


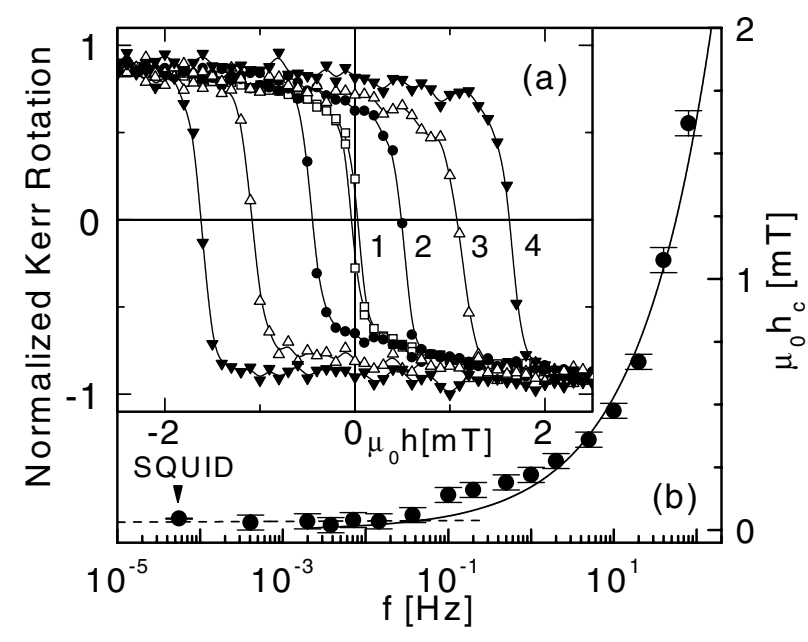

FIG. 4. (a) Normalized MOKE loops obtained at $T=294 \mathrm{~K}$ and $f=0.002$ (1), 0.1 (2), 10 (3), and $40 \mathrm{~Hz}$ (4), and (b) $f$ dependence of the coercive field including the quasistatic SQUID result, best fitted to power laws $h_{c} \propto f^{\alpha}$ with $\alpha(f<$ $0.1 \mathrm{~Hz})=0.02$ and $\alpha(f>1 \mathrm{~Hz})=0.49$ (dashed and solid lines, respectively; see text).

broken and solid line, respectively). Different domain processes, viz. wall motion and domain nucleation, respectively, are supposed to dominate these regimes [12]. While the exponents observed in our SFM sample are very similar to those of continuous ferromagnetic films [12], the magnetic field sweep rates at the crossover from the "low" to the "high dynamic regime" are by about 3 orders of magnitude smaller. This clearly characterizes the extreme softness of our dipolarly coupled SFM system.

The above considerations of quasistatic and dynamic hysteresis loops help interpreting the ac susceptometric results (Figs. 1 and 2), since $\chi^{*}$ is related to the field derivative of the nonlinear function $M(h)$ by averaging over partial loops. Owing to the dynamics of $M(h)$, one has to account for its dependence on both $T$ (Fig. 3 ) and $f$ (Fig. 4). Hence, for low $T$ and/or high $f$, a weak field $h_{0}$ probes relaxation and thermally activated creep of the domain walls [13] in the central part of the hysteresis loop, while for high $T$ and/or low $f$, complete magnetization switching might be accomplished by the same field amplitude $h_{0}$. This explains the difference between the lower and upper parts of the Cole-Cole diagrams in Fig. 2(a) as will be explained in the following.

At very low $h_{0}$ and sufficiently high $f$, the spectra are observed to start as horizontal lines [Fig. 2(b) for $\mu_{0} h_{0}=$ $5 \mu \mathrm{T}]$. Obviously, in this limit the response has the appearance of "white noise." It corresponds to a constant power spectrum (via the fluctuation-dissipation theorem) and is compatible to a power-law dependence $\chi^{\prime} \propto \omega^{-\beta}$ as confirmed experimentally [14]. This behavior is typical of the reversible relaxation of pinned domain wall segments with a quasicontinuous distribution of Debye-type spectra [15].
This spectral behavior changes abruptly at a threshold frequency denoted by $h_{\omega}$ [Fig. 2(b)], where the horizontal line bends up to a finite slope, such that $\chi^{\prime \prime} \propto \chi^{\prime}$. Here the predicted [7] transition from the reversible relaxation into the irreversible creep regime is observed for the first time. It occurs at all of our $h_{0}$ values as shown in Fig. 5 by the respective phase lines denoted as " $h_{\omega}=5,50$, and 200." The temperature/frequency regions at $T<T\left(h_{\omega}\right)$ and $\omega>\omega\left(h_{\omega}\right)$ thus refer to reversible wall relaxation regimes being large for " $h_{\omega}=5$," but very restricted for " $h_{\omega}=200 . "$

In order to understand the linear relationship $\chi^{\prime \prime} \propto \chi^{\prime}$ observed in the creep regime for $\omega<\omega\left(h_{\omega}\right)$ [Figs. 2(a) and 2(b); high- $f$ side], let us first consider viscous domain wall motion in the sliding regime [13]. In a simple stripe domain model related to up and down domains with initial uniform width $d$ and magnetization $\pm M_{s}$, the sideways motion of their walls along the coordinate $x$ under an external field $h(t)$ yields the rate equation,

$$
d M / d t=\left(2 M_{s} / d\right) \mu_{w} h(t),
$$

where the wall mobility $\mu_{w}$ and $h(t)$ determine the wall velocity, $d x / d t=\mu_{w} h(t)$. Assuming constant $\mu_{w}$ at weak fields (above the depinning threshold [13]), one finds a linear time dependence of the magnetization, $M(t)=\left(2 \mu_{w} M_{s} / d\right) H t$, in a constant field $H$, while a harmonic one, $h(t)=h_{0} \exp (i \omega t)$, yields

$$
M(t)=\left(2 \mu_{w} M_{s} / i \omega d+\chi_{\infty}\right) h_{0} \exp (i \omega t) .
$$

Here, the second term refers to the "instantaneous" reversible domain wall response occurring on shorter time scales (see above). Weak periodic fields thus probe a linear ac susceptibility $\chi^{*}=\chi_{\infty}\left[1+1 /\left(i \omega \tau_{i}\right)\right]$ with $\chi_{\infty} / \tau_{i} \equiv$ $\left(2 \mu_{w} M_{s} / d\right)$, where $\tau_{i}$ denotes the time, in which the interface contribution to the magnetization equals the instantaneous one, $\Delta M=\chi_{\infty} h$.

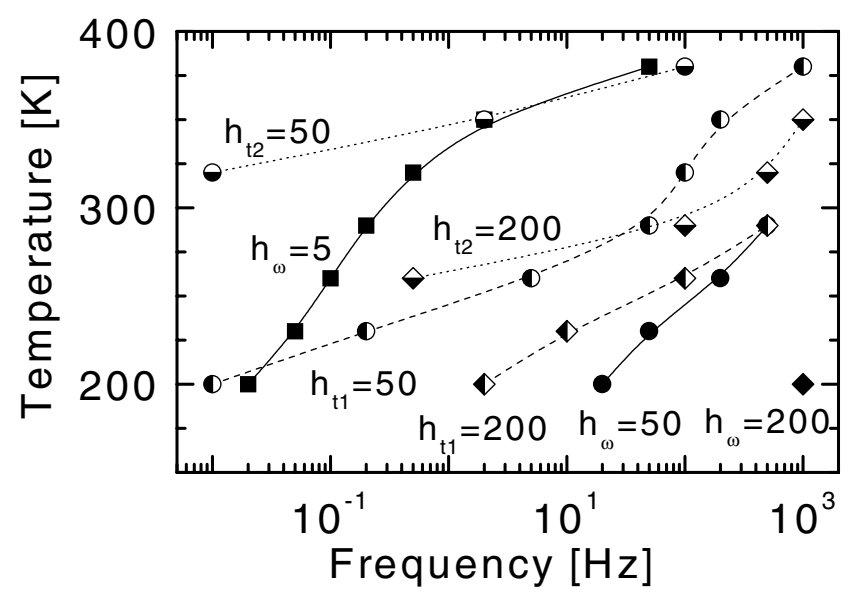

FIG. 5. Dynamical phase transition lines $h_{\omega}, h_{t 1}$, and $h_{t 2}$ in the $T$ - $f$ plane determined from Cole-Cole plots for amplitudes $\mu_{0} h_{0}=5,50$, and $200 \mu \mathrm{T}$, respectively, (see text). 
In the next step, we have to account for the nonlinearity of $v$ vs $h$ in the creep regime of thermally excited viscous motion, $h_{\omega}<h<h_{t 1}$ (= depinning field) [13]. Approximating this regime roughly by $v \propto h^{\delta}, \delta>1, \chi^{*}$ may be modified phenomenologically by introducing an exponent $\beta<1$ and an effective relaxation time $\tau_{\text {eff }}$,

$$
\chi^{*}=\chi_{\infty}\left[1+1 /\left(i \omega \tau_{\text {eff }}\right)^{\beta}\right],
$$

similarly as used in the case of polydispersive Debyetype relaxation [10].

Decomposing Eq. (3) into $\chi^{\prime}=\chi_{\infty}[1+\cos (\beta \pi / 2) /$ $\left.\left(\omega \tau_{\text {eff }}\right)^{\beta}\right]$ and $\chi^{\prime \prime}=\chi_{\infty} \sin (\beta \pi / 2) /\left(\omega \tau_{\text {eff }}\right)^{\beta}$ then yields the linear relationship $\chi^{\prime \prime} /\left(\chi^{\prime}-\chi_{\infty}\right)=\tan (\pi \beta / 2)$, which is observed in our experiments (Fig. 2). Inspection of numerous spectral data $\chi^{\prime}$ and $\chi^{\prime \prime}$ vs $\log \omega$ [14] reveals power laws with exponents as low as $\beta \approx$ $0.1-0.2$, which may be interpreted to be due to a wide distribution of $\mu_{w}$.

Upon decreasing $\omega$ at a given $T$ and $h_{0}$, the linearity $\chi^{\prime \prime} \propto \chi^{\prime}$ does no longer hold above a threshold value $\omega_{t 1}$ [Fig. 2(a); arrow designated as $h_{t 1}$ ]. Tentatively, we assume that the sliding regime is entered above this depinning threshold, where $\chi^{\prime \prime}$ is expected to rise very steeply under the idealized conditions of a stripe domain model with equidistant domains (see above). Hence, the end point of the linear regime provides a criterion for the transition expected at $h_{t 1}$ [7]. Again, we have checked numerous data sets $\chi^{\prime \prime}$ vs $\chi^{\prime}[14]$ and thus constructed the phase lines referring to $\mu_{0} h_{t 1}=50$ and $200 \mu \mathrm{T}$ in Fig. 5 . Consequently, the regions between the lines $h_{t 1}$ and $h_{t 2}$, i.e., for $T\left(h_{t 2}\right)>T>T\left(h_{t 1}\right)$ and $\omega_{t 2}<\omega<\omega_{t 1}$, comply with the sliding condition for the respective value of $h_{0}$.

Complete switching experienced by ac susceptometry corresponds to Avrami-Fatuzzo-type nucleation and growth processes [16] approximately obeying first-order rate equations:

$$
d M / d t=\tau^{-1}\left[ \pm M_{s}-M(t)\right],
$$

with solutions $M(t)= \pm M_{s}[1-2 \exp (-t / \tau)]$, where alternative signs hold for consecutive half periods. $\pm M_{s}$ and $\tau$ are the saturation magnetization and an averaged relaxation time, respectively. The corresponding susceptibility in the linear response regime is Debye-type, $\chi^{*}=$ $\chi_{\infty} /(1+i \omega \tau)$, and yields a perfect Cole-Cole semicircle, $\chi^{\prime \prime}$ vs $\chi^{\prime}$ [10]. Hence, the broken circles fitted to curves 1 and 2 in Fig. 2(a) refer to those frequencies, at which the domain wall proceeds from one sample boundary to the other in the dynamic single domain wall model [7]. According to this criterion, we have determined various upper frequencies corresponding to $\mu_{0} h_{0} \equiv h_{t 2}=50$ [Fig. 2(a)] and $200 \mu \mathrm{T}$ [14] and plotted versus $T$ in Fig. 5 (dotted curves). Complementarily to the transition lines in the $T$ vs $h$ phase diagram [7], the regions [ $T>$ $T\left(h_{t 2}\right), \omega<\omega_{t 2}$ ] refer to "full switching." It will be interesting to test the expected [7] sample size dependence of the critical lines $h_{t 2}$ vs $T$.

In conclusion, the ac susceptibility measured on SFM $\left[\mathrm{Co}_{80} \mathrm{Fe}_{20}(1.4 \mathrm{~nm}) / \mathrm{Al}_{2} \mathrm{O}_{3}(3 \mathrm{~nm})\right]_{10}$ in different frequency and field regimes reveals for the first time the phase transitions predicted for the hysteretic dynamics of pinning-controlled domain wall motion [7]. The linear responses of wall relaxation [15], creep and sliding wall motion, and Avrami-Fatuzzo-type switching, respectively, have been described phenomenologically. A systematic transcription of the hysteresis equations [7] into those of the linear response (by appropriate Fourier transformation) has still to be accomplished. Experimentally, it remains a challenge to discover the predicted phase transitions via direct $M$ vs $h$ measurements, and to image the domain walls and their field-induced displacement, e.g., by scanning magnetic force microscopy.

We thank S. Sahoo for valuable discussions and the DFG (Graduate School "Structure and Dynamics of Hetrogeneous Systems" and SFB 491) for financial support.

[1] W. Kleemann et al., Phys. Rev. B 63, 134423 (2001).

[2] G. N. Kakazei et al., J. Appl. Phys. 90, 4044 (2001).

[3] U. Bovensiepen et al., J. Magn. Magn. Mater. 192, L386 (1999).

[4] P. J. Jensen and G. M. Pastor, Phys. Status Solidi (a) 189, 527 (2002).

[5] V. N. Kondratyev and H. O. Lutz, Phys. Rev. Lett. 81, 4508 (1998); P. Bruno, ibid. 88, 240401 (2002); R. V. Chamberlin (unpublished).

[6] J. Villain, Z. Phys. B 33, 31 (1979); C. L. Henley, Phys. Rev. Lett. 62, 2056 (1989).

[7] T. Nattermann, V. Pokrovsky, and V. M. Vinokur, Phys. Rev. Lett. 87, 197005 (2001).

[8] Ch. Morawe and H. Zabel, J. Appl. Phys. 77, 1969 (1995); L. F. Schelp et al., Phys. Rev. B 56, R5747 (1997).

[9] O. Petracic et al., Phase Transit. 75, 73 (2002); S. Sahoo et al., Phys. Rev. B 65, 134406 (2002).

[10] A. K. Jonscher, Dielectric Relaxation in Solids (Chelsea Dielectrics Press, London, 1983).

[11] M. Acharyya and B. K. Chakrabarti, Phys. Rev. B 52, 6550 (1995); F. Zhong and J. X. Zhang, Phys. Rev. Lett. 75, 2027 (1995).

[12] W. Y. Lee et al., J. Appl. Phys. 87, 5926 (2000).

[13] M.V. Feigel'man et al., Phys. Rev. Lett. 63, 2303 (1989); S. Lemerle et al., ibid. 80, 849 (1998).

[14] O. Sichelschmidt, Diploma thesis, Gerhard-MercatorUniversität Duisburg, 2002.

[15] L. B. Ioffe and V. M. Vinokur, J. Phys. C 20, 6149 (1987); T. Nattermann, Y. Shapir, and I. Vilfan, Phys. Rev. B 42, 8577 (1990).

[16] M. Avrami, J. Chem. Phys. 7, 1103 (1939); E. Fatuzzo, Phys. Rev. 127, 1999 (1962). 